\title{
Niveles de mercurio y percepción del riesgo en una población minera aurífera del Guainía (Orinoquia colombiana)
}

\author{
Alvaro J. Idrovo ${ }^{1}$, Luis E. Manotas ${ }^{2}$, Gladys Villamil de García ${ }^{3}$, Jaime E. Ortiz ${ }^{3}$, \\ Elizabeth Silva $^{3}$, Saúl A. Romero ${ }^{2}$, Carlos E. Azcárate ${ }^{2}$ \\ ${ }^{1}$ Departamento de Salud Pública y Tropical, Facultad de Medicina, Universidad Nacional de Colombia, \\ Bogotá, D.C., Colombia. \\ ${ }^{2}$ Secretaría de Salud del Guainía, Guainía, Colombia. \\ ${ }^{3}$ Laboratorio de Salud Ambiental, Instituto Nacional de Salud, Bogotá, D.C., Colombia.
}

La minería aurífera en la región de la Orinoquia colombiana ha tenido un importante crecimiento en los últimos 15 años. Esta actividad se ha convertido en un importante problema de salud pública debido a varios factores como el uso de mercurio durante el proceso de amalgamación del oro, la contaminación del ecosistema y la exposición de la población de la región. El objetivo del presente trabajo fue determinar los niveles de mercurio en cabello y sangre en una población minera del departamento del Guainía y la percepción que tiene dicha población del riesgo de usar este elemento. Para tal fin, se encuestaron 78 residentes de la región, de los cuales se obtuvieron 59 muestras de sangre y 61 de cabello.

Los resultados indican un precario ambiente laboral y una falta de conocimiento del riesgo ecológico, lo cual permite la contaminación del ecosistema de la Orinoquia. Los individuos perciben la exposición al mercurio como nociva para la salud, aunque sus conocimientos al respecto son inadecuados. Los valores de mercurio en sangre entre los mineros oscilaron entre 6,9 y $168,0 \mu \mathrm{g} / \mathrm{l}$ (promedio=59,16) y entre los individuos indirectamente expuestos fluctuó entre 17,7 y $100,8 \mu \mathrm{g} / \mathrm{l}$ (promedio=53,5). Las concentraciones de mercurio total en cabello oscilaron entre 3,0 y $89,2 \mu \mathrm{g} / \mathrm{g}$ (promedio=26,93) en el grupo de mineros y entre 2,8 y $48,7 \mu \mathrm{g} / \mathrm{g}$ (promedio $=22,86$ ) entre los expuestos indirectamente. No se encontraron diferencias significativas en los niveles de mercurio entre los dos grupos estudiados. Se sugieren medidas preventivas y de control de la situación generada por el uso de mercurio en las labores de minería aurífera de la región.

Palabras clave: mercurio, minería aurífera, percepción del riesgo, Orinoquia, Colombia.

Mercury levels and risk perception in a gold mining population from Guainía, Colombia (Orinoquia region)

Gold mining in the Colombian Orinoquia region had a fast growth in the last 15 years. Mercury is used during the gold amalgam process, and its presence is an important problem of public health because it produces a polluted ecosystem and increases the risk of mercury-related adverse health effects among the exposed human population. The main objective of this study was to determine the mercury levels in blood and hair, and the risk perception of the mercury exposure in a population from Guainía. Fifty-nine blood and sixty-one hair samples were collected to measure the mercury concentration.

The findings show a very deteriorated occupational environment that results in contamination of the Orinoquia ecosystem. The subjects perceived the mercury exposure like noxious to their health, although their knowledge about mercury-related health effects is inadequate. The mercury levels in blood are between 6.9 and $168.0 \mu \mathrm{g} / \mathrm{l}$ (mean=59.16) among the mining workers, and between 17.7 y $100.8 \mu \mathrm{g} / \mathrm{l}$ (mean=53.50) among subjects indirectly exposed. The total mercury levels in hair are between 3.0 and $89.2 \mu \mathrm{d} / \mathrm{g}$ (mean=26.93) among the mining workers, and from 2.8 to $48.70 \mu \mathrm{g} / \mathrm{g}$ (mean=22.86) among subjects indirectly exposed. No significant differences were found between the two exposure groups studied. We suggest preventive and 
control measures to minimize the exposure to mercury related with gold mining in the region under study.

Key words: mercury, gold mining, risk perception, Orinoquia, Colombia.

El mercurio metálico se utiliza en las actividades de extracción de oro tanto aluvial como de filón. En el proceso de la extracción aluvial, el mercurio forma una amalgama con el oro granulométrico que se encuentra disperso en el sedimento del lecho del río; posteriormente, el mercurio puede ser lanzado al ambiente en forma de vapor durante la quema de la amalgama y en forma líquida a través de los vertimientos que son arrojados a los cuerpos de agua o que se dejan a campo abierto sin ningún tipo de tratamiento.

El vapor del mercurio puede ser inhalado por el hombre, en cuyo caso es retenido en un $80 \%$ y oxidado rápidamente a ion mercúrico en la sangre y los tejidos (1), o puede oxidarse y regresar al ambiente acuático donde los microorganismos que viven en los sedimentos pueden transformar el mercurio inorgánico en metilmercurio, el cual es acumulado y movilizado en las cadenas alimenticias acuáticas, llegando posteriormente al hombre. Como efectos de la exposición aguda al mercurio inorgánico, se pueden presentar bronquitis o bronquiolitis erosiva con neumonitis intersticial. Por exposición crónica a bajos niveles, se pueden observar debilidad, fatiga, anorexia y disturbios gastrointestinales (micromercurialismo); el temblor en dedos, párpados y labios constituye el signo clásico de la exposición crónica. Este último puede acompañarse de efectos sobre el sistema nervioso central como pérdida de la memoria, insomnio, excitabilidad, depresión y disturbios leves o súbitos de la personalidad (2).

Debido a informes recibidos en el Laboratorio de Salud Ambiental del Instituto Nacional de Salud (LSA-INS) desde mediados de la década de los 80 , la contaminación ambiental puede estar ocurriendo en la región de la Orinoquia colombiana. Por ejemplo, se sabe que en 1992 estaban

Correspondencia:

Elizabeth Silva

esilva@hemagogus.ins.gov.co

Recibido: 21/12/00; aceptado: 04/05/01 operando 13 barcazas flotantes y el consumo de mercurio se calculó en 30 kg/mes. En 1994, subió el número de barcazas a 25 y el consumo de mercurio a $50 \mathrm{~kg} / \mathrm{mes}$. Todos los desechos del proceso de la extracción del oro eran vertidos en forma directa al ambiente. El quemado de la amalgama se hacía a la intemperie; por tanto, la emanación de los vapores mercuriales a la atmósfera era total y los desechos de agua y arena se dejaban a campo abierto (3). Para 1998, la Secretaría de Salud del Guainía conocía de la presencia de 14 barcazas en la región y de la existencia de una tendencia en el desplazamiento de la actividad minera desde el río Inírida hacia el río Guainía, perteneciente a la cuenca amazónica.

Ante esta situación, el objetivo del presente estudio fue determinar los valores de mercurio en sangre y cabello en un grupo de individuos que trabajan o tienen relación directa con la extracción de oro en la región del Guainía, así como describir el ambiente en el que trabajan los mineros, la sintomatología probablemente asociada con la exposición, la percepción que ellos tienen del riesgo de exposición al mercurio y su conocimiento acerca de los posibles efectos adversos en la salud. Con esta aproximación, se pretende explorar la situación general de la problemática para poder determinar las actividades dirigidas a prevenir la aparición de efectos adversos sobre la salud de los residentes en dicha región.

\section{Materiales y métodos}

\section{Población y área de estudio}

Entre octubre de 1998 y enero de 1999, se realizó un estudio descriptivo exploratorio en la cuenca hidrográfica del río Inírida en la zona comprendida entre el caserío Buenavista-Chorrobocón y la desembocadura del caño Tonina (figura 1), incluidas las localidades de Cerro Nariz, Zamuro y Chorrobocón ( $3^{\circ} 52^{\prime}$ latitud norte y $67^{\circ} 55^{\prime}$ longitud oeste). Esta región corresponde a una zona geográfica del suroriente de Colombia, con una población aproximada de 480 habitantes, cuya 


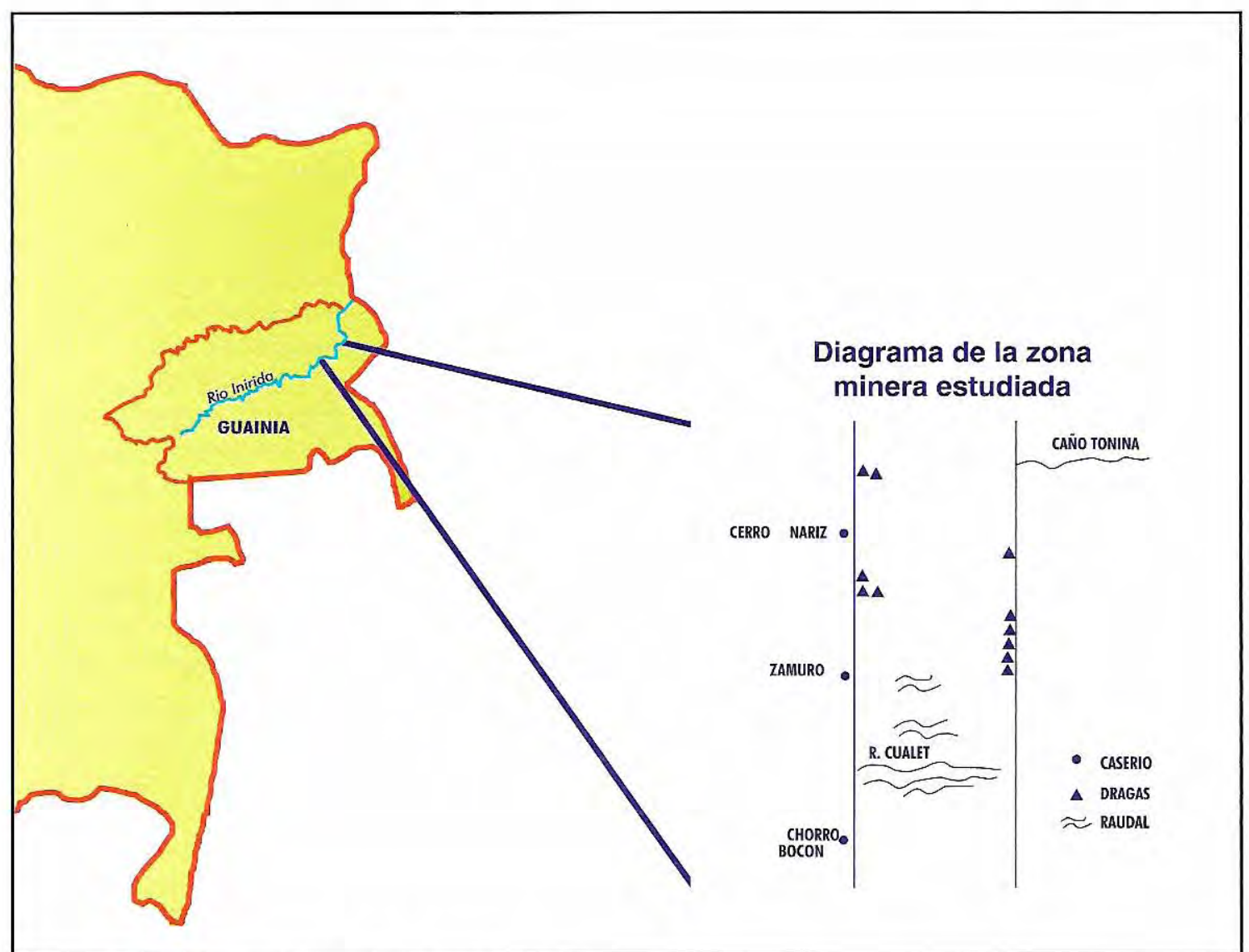

Figura 1. Localización geográfica de la zona estudiada.

actividad económica principal es la explotación de oro de aluvión, empleando mercurio para el proceso de amalgamación en forma artesanal y sin ninguna reglamentación. En toda la cuenca del río Inírida, se encuentran aproximadamente 1.700 habitantes que tienen condiciones similares.

La cercanía de la zona a Puerto Inírida fue el criterio clásico para la selección de la población debido a las dificultades logísticas para realizar un estudio en las extensas áreas de la Orinoquia. Dentro de la región, los individuos invitados a participar debían tener como características el ser residente en la zona, tener algún tipo de relación con las labores de minería aurífera y haber incluido en su dieta pescado y agua de la zona de estudio durante los 5 años previos; en todos los casos, la participación fue voluntaria.

El trabajo tuvo dos componentes: 1) una encuesta de conocimientos y percepción del riesgo asociado a la exposición al mercurio y 2) la cuantificación de mercurio total en muestras biológicas en la población estudiada. En la encuesta, se les interrogó acerca de la edad, el género, la ocupación, los hábitos alimenticios, las medidas de control de la exposición, los posibles síntomas asociados con la contaminación por mercurio y los conocimientos acerca de la forma de exposición al mercurio y de sus potenciales efectos adversos. Para el análisis de los datos, se consideró como grupo expuesto a todos aquellos individuos directamente involucrados en las labores de minería y, como indirectamente expuestos, a los familiares de los mineros o a aquellos sujetos que se encontraban realizando otro tipo de actividades en el mismo caserío.

Para el componente de cuantificación de mercurio en muestras biológicas, se recolectaron 65 muestras de sangre y 66 de cabello; esta diferencia 
se debe a que hubo individuos que sólo permitieron la toma de una de las muestras. Las muestras de cabello se recolectaron cortando un mechón de pelo lo más cercano posible al cuero cabelludo; posteriormente se fijaron con cinta adhesiva a una hoja de papel, indicando el segmento proximal y el distal, y se depositaron individualmente en un sobre de manila. Las muestras de sangre fueron recolectadas en tubos vacutainer estériles con EDTA; posteriormente, fueron enviadas por vía aérea al laboratorio en neveras de icopor, con bolsas de agua congelada para mantener una temperatura menor a $6^{\circ} \mathrm{C}$. Una vez las muestras ingresaron al LSA-INS, se conservaron a $4{ }^{\circ} \mathrm{C}$ hasta la realización del análisis.

\section{Método analítico}

La determinación de mercurio total en cabello y sangre se realizó en el laboratorio de espectrofotometría del LSA-INS, por el método de Lefebvre y colaboradores modificado en el LSAINS, empleando el equipo de absorción atómicavapor frío MAS-50 Coleman (4). Las muestras de cabello se lavaron y, posteriormente, fueron sometidas a digestión con adición de ácido nítrico y a alta presión y temperatura en un horno microondas.

El rango lineal de trabajo hallado experimentalmente en el laboratorio para mercurio en cabello fue de $0,3 \mathrm{a} 20 \mu \mathrm{g} / \mathrm{g}$ y para mercurio en sangre de 1,7 a $30 \mu \mathrm{g} / \mathrm{l}$. Para el control de calidad interno en sangre, se utilizaron muestras certificadas de $5 \mathrm{y}$ $32,09 \mu \mathrm{g} / \mathrm{l}$ enviadas por el Centro Toxicológico de Quebec (CTQ); la desviación estándar en las 10 corridas fue de 0,95 y $1,77 \mu \mathrm{g} / \mathrm{l}$, respectivamente. El control de calidad interno en cabello se realizó con las muestras certificadas de 7,4 y $17,1 \mu \mathrm{g} / \mathrm{g}$ proporcionadas por el Centro Toxicológico de Ottawa-Canadá (CTOC); la desviación estándar en las 15 corridas fue 0,52 y $0,74 \mu \mathrm{g} / \mathrm{g}$, respectivamente. El control de calidad externo se basa en la participación en los programas interlaboratorios del CTQ y el CTOC.

\section{Métodos estadísticos}

En la primera parte del trabajo, se realizó la descripción de la población estudiada, su percepción del riesgo de exposición al mercurio, sus conocimientos acerca de los potenciales efectos adversos del mercurio y del ambiente laboral según el nivel de exposición. Para explorar las diferencias entre los grupos, se utilizaron las pruebas de $x^{2}$ y exacta de Fisher según la forma de presentación de las variables. Los niveles de mercurio en sangre y cabello entre el grupo de trabajadores mineros y el grupo de individuos expuestos indirectamente al mercurio se compararon mediante la prueba de Mann-Whitney. En todos los casos, se estableció $p<0,05$ como nivel de significación estadística.

\section{Resultados}

La población estudiada se encuentra entre los 13 y los 63 años de edad (promedio=31,78 años; desviación estándar=11,04); en el grupo de mineros, la edad promedio fue de 34,19 años (desviación estándar=10,90), con valores que oscilaron entre 18 y 63 años; en el grupo de expuestos indirectamente al mercurio, el promedio fue de 28,34 años (desviación estándar=10,51), con valor mínimo de 13 y mayor de 59 años. Otras características de la población estudiada se encuentran en el cuadro 1. Como se puede apreciar, existen notables diferencias en la composición de los dos grupos. Este hecho se puede explicar por la circunstancia de que el grupo de mineros está constituido por hombres de raza blanca que, debido a las características laborales, trabajan sin un horario predeterminado. El grupo expuesto indirectamente se caracteriza por estar conformado por familiares de los mineros (esposas e hijos).

El ambiente laboral de estos individuos es muy precario. El tipo de azogadero más frecuente es el individual $(89,19 \%)$. El lugar donde realizan al amalgamamiento generalmente es al aire libre $(83,78 \%)$ y sólo en el $16,22 \%$ de los casos se encuentra un extractor de vapores, que los mineros no suelen utilizar. Unicamente el $13,51 \%$ refiere el uso de una mesa especial para la realización de su trabajo. Los elementos de protección personal también son escasos; sólo el $8,11 \%$ dice usar guantes; el $16,22 \%$, tapabocas, y el $5,56 \%$, blusa de trabajo. La gran mayoría de los trabajadores (80\%) refiere que lava la ropa de trabajo en el propio río donde trabajan. Los 
Cuadro 1. Características de la población estudiada.

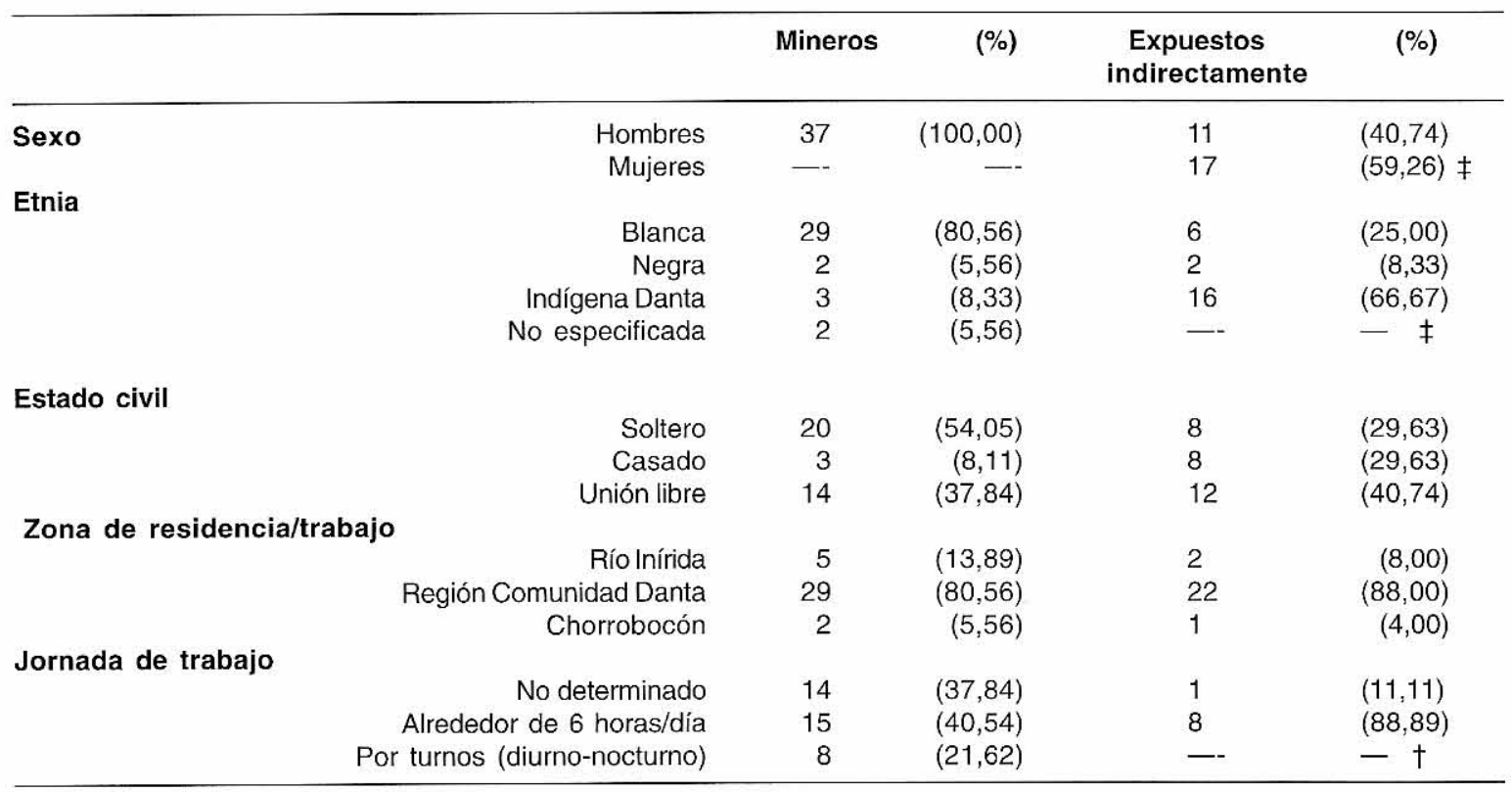

$\dagger: p<0,05 ; \ddagger: p<0,01$

individuos encuestados informan en un $85,71 \%$ que los desechos de mercurio son enterrados; sin embargo, sólo el $40 \%$ informa que los desechos con mercurio son almacenados en envases plásticos antes de ser enterrados, lo que permite suponer que el mercurio se está dejando libremente en el suelo en un gran número de ocasiones. Según los encuestados, las principales formas de evitar la intoxicación con mercurio es mediante el uso de máscaras protectoras $(29,09 \%)$, utilizando hornos durante el azogue $(27,27 \%)$ o usando guantes y tapabocas $(20 \%)$.

El riesgo de exposición es alto, tanto para los propios mineros como para sus familiares debido a que el $22 \%$ informa que su familia se encuentra cerca al lugar de trabajo y el $61,11 \%$ toma los alimentos en el lugar de trabajo. La percepción y conocimiento que tiene esta población del riesgo asociado con la exposición al mercurio es muy limitada y, en algunos casos, incorrecta. EI $77,19 \%$ considera que el mercurio es un metal, mientras que los demás lo consideran un gas $(10,53 \%)$ o una sustancia no metálica $(12,28 \%)$ que se encuentra únicamente en sus manos y ropa de trabajo $(22,41 \%)$, en el aire, el suelo y las fuentes de agua $(18,97 \%)$, o en todas las anteriores $(56,90 \%)$; muy pocos $(1,72 \%)$ saben que puede encontrarse en los pescados, mariscos y vegetales de la región. El $65,52 \%$ de los individuos considera como principal vía de entrada del mercurio al organismo la vía respiratoria y digestiva. Pese a estos hallazgos, casi la totalidad de los encuestados $(94,92 \%)$ considera el mercurio como un elemento potencialmente nocivo para su salud, que afecta principalmente el sistema nervioso y los órganos de la visión (54,55\%). El $82,29 \%$ considera el mercurio como teratógeno.

Como se puede apreciar en los cuadros 2 y 3 , los valores encontrados en cabello en la población tienen un rango amplio que varía entre 2,8 y 89,2 $\mu \mathrm{g} / \mathrm{g}$ (figura 2); lo mismo ocurre para las concentraciones de mercurio en sangre, donde se encuentran valores que oscilan entre 6,9 y 168 $\mu g / l$ (figura 3). Dado que la concentración de mercurio es cerca de 300 veces más alta en cabello que en sangre (5), se realizó la comparación de los valores obtenidos en los dos tipos de matrices. Para esto, se utilizó el programa Kalibo (6) y mediante regresión lineal de primer orden se encontró una pendiente de 345, cercana a 300 , que era lo esperado. Sólo el $4,6 \%$ de los individuos presentan concentraciones de mercurio 
Cuadro 2. Niveles de mercurio en sangre según grupos estudiados.

\begin{tabular}{lcccccc}
\hline & \multicolumn{7}{c}{ Sangre $(\boldsymbol{\mu g} / \mathbf{l})$} \\
\cline { 2 - 7 } & $\mathbf{n}$ & Mínimo & Máximo & Promedio & DE & Mediana \\
\hline Mineros & 34 & 6,90 & 168,00 & 59,16 & 32,17 & 52,65 \\
Expuestos indirectamente & 25 & 17,70 & 100,80 & 53,50 & 20,02 & 54,10 \\
\hline
\end{tabular}

Valores aceptables en sangre: $<20 \mu \mathrm{g} / \mathrm{l}$

Cuadro 3. Niveles de mercurio en cabello según grupos estudiados.

\begin{tabular}{lcccccc}
\hline & \multicolumn{5}{c}{ Cabello $(\boldsymbol{\mu g} / \mathbf{g})$} \\
\cline { 2 - 6 } & $\mathbf{n}$ & Mínimo & Máximo & Promedio & DE & Mediana \\
\hline Mineros & 39 & 3,00 & 89,20 & 26,93 & 19,07 & 20,50 \\
Expuestos indirectamente & 22 & 2,80 & 48,70 & 22,86 & 13,81 & 21,40 \\
\hline
\end{tabular}

Valores aceptables en cabello: $<5 \mu \mathrm{g} / \mathrm{g}$

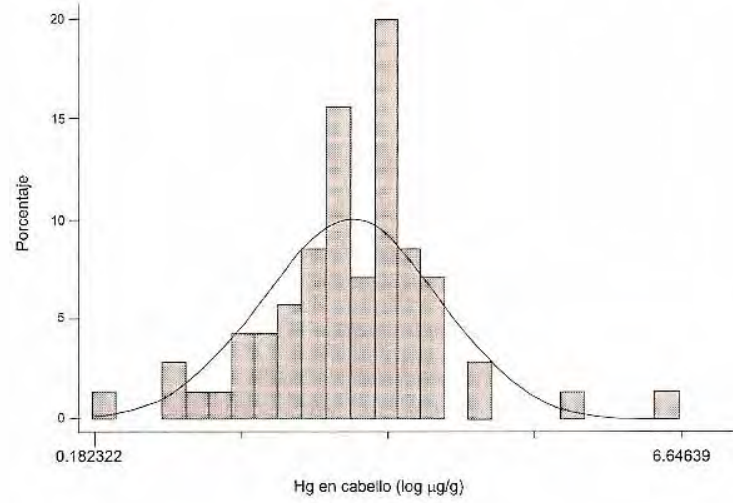

Figura 2. Niveles de mercurio en cabello en la población estudiada.

por debajo del límite máximo permisible. Los síntomas presentados en la población por encima de los valores aceptables (sangre: $<20 \mu \mathrm{g} / \mathrm{l}$ y cabello: $<5 \mu \mathrm{g} / \mathrm{g}$ ), se encuentran en el cuadro 4 . Allí se puede apreciar que los síntomas más prevalentes son la pérdida de peso, el cansancio físico, la disminución de la productividad en el trabajo, la disminución de la memoria, el temblor y el insomnio. Sin embargo, estos síntomas no se correlacionan con los niveles de mercurio en sangre o cabello.

Al comparar los valores de mercurio en sangre entre las dos poblaciones no se encuentran diferencias significativas ( $p=0,7185)$; este hecho se repite cuando se comparan los valores de

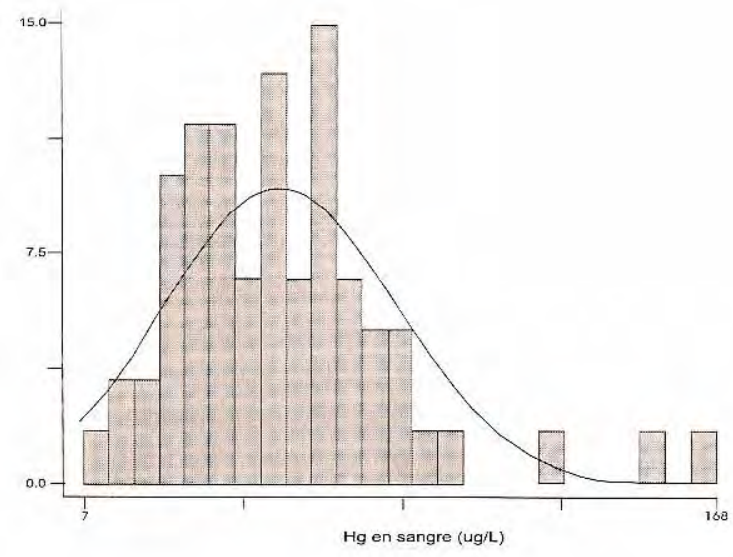

Figura 3. Niveles de mercurio en sangre en la población estudiada.

mercurio en cabello ( $p=0,5430)$. Estos hallazgos deben entenderse en el sentido de que todos los individuos de la población estudiada se encuentran expuestos al mercurio y que la actividad realizada por estos sujetos no determina las concentraciones de metal en las matrices estudiadas.

\section{Discusión}

Este trabajo debe entenderse como una primera aproximación a la problemática sanitaria asociada al uso de mercurio en las labores de minería aurífera en la Orinoquia colombiana. Situaciones similares se presentan en otras regiones de Colombia, entre las que deben destacarse los departamentos de Chocó, Bolívar, Santander y Caldas (7-10), así como en otros países como 
Cuadro 4. Porcentaje de individuos con síntomas según concentraciones de mercurio superiores a los valores aceptables.

\begin{tabular}{lcc}
\hline \multicolumn{1}{c}{ Síntomas } & Cabello>5 $\boldsymbol{\mu \mathbf { g } / \mathbf { g }}$ & Sangre>20 $\boldsymbol{\mu g} / \mathbf{l}$ \\
\hline Sabor metálico & 8,77 & 8,62 \\
Sensación de quemadura & 7,02 & 6,90 \\
Sangrado de encías & 8,77 & 8,62 \\
Salivación excesiva & 19,30 & 18,97 \\
Disminución de agudeza visual & 17,54 & 17,24 \\
Pérdida de piezas dentarias & 14,04 & 15,52 \\
Pérdida de apetito/peso & 26,32 & 27,59 \\
Cansancio & 43,86 & 44,83 \\
Vómito o diarrea & 10,53 & 10,34 \\
Disminución de productividad laboral & 40,43 & 38,78 \\
Disminución de la memoria & 33,33 & 32,00 \\
Temblor & 39,58 & 38,00 \\
Insomnio & 33,33 & 32,00 \\
Ansiedad & 33,33 & 32,00 \\
Delirios & 6,25 & 6,00 \\
Desconfianza & 14,58 & 14,00 \\
\hline
\end{tabular}

Brasil, Venezuela, Perú, Bolivia, Guayana y Surinam (11-17). Los hallazgos más importantes se refieren a las precarias condiciones de trabajo de la población, que se relacionan directamente con la extracción de oro en la región de la Orinoquia colombiana, así como de los habitantes de la zona, además de la presencia de población de alto riesgo, niños y mujeres, cerca del lugar de quema de la amalgama. Al comparar los valores encontrados en este estudio con las concentraciones de mercurio en cabello descritas entre individuos de los ríos Madeira $(0,22-303 \mu \mathrm{g} / \mathrm{g})$, Tapajós $(0,7-142 \mu \mathrm{g} / \mathrm{g}$ ) y Negro (promedio $=75,5$ $\mu \mathrm{g} / \mathrm{g})$, y en las represas de Tucurí $(0,9-240 \mu \mathrm{g} / \mathrm{g})$ y Balbina en Brasil (1,15-26 $\mu \mathrm{g} / \mathrm{g})$ (15), país donde se han realizado más estudios al respecto, los valores encontrados en el Guainía sólo son superados en las regiones del río Negro y el embalse Tucurí.

Pese a la alta absorción de mercurio descrita en este trabajo, en Colombia aún son muy pocos los estudios acerca de la contaminación con mercurio y los efectos adversos sobre la salud asociados con la exposición a este metal. Aunque la información aquí presentada no es representativa y conclusiva de la totalidad de la población minera y residente en la Orinoquia colombiana, puede servir como indicio de una probable amenaza para la salud de estas poblaciones. Al comparar los hallazgos aquí presentados con los estudios realizados por Olivero y colaboradores (18-20) en la zona norte del país, se evidencia que el peligro es mayor en la región de la Orinoquia, quizás debido a la magnitud del fenómeno social en esta amplia zona del país.

Un problema no abordado en el presente trabajo, que podría estar sucediendo en la región estudiada, es la transformación de la forma inorgánica del mercurio a compuestos orgánicos (metil y etilmercurio, principalmente) (21). Estas formas químicas, en especial la primera, son más tóxicas para los humanos que las formas elementales; la mayoría de los efectos observados se presentan sobre el sistema nervioso e incluyen trastornos motores y sensitivos, alteraciones mentales (retraso psicomotor y daños neurológicos tardíos) y nefrotoxicidad. Cuando la exposición es prenatal, mediante el paso del compuesto a través de la barrera placentaria, se pueden presentar efectos neurológicos severos con trastornos motores y sensitivos, microcefalia, ataxia, temblores, disartria y ceguera $(1,22)$.

La situación ambiental y de salud descrita en el presente trabajo constituye un gran reto para la vigilancia en salud pública (23). Las actividades mínimas sugeridas para el manejo y control de esta situación deben incluir la caracterización demográfica de la población asociada a los centros mineros, la determinación de los niveles de absorción del mercurio en los habitantes de la región, diferenciando los compuestos orgánicos de los inorgánicos, la promoción del uso de 
equipos que minimicen las emisiones de mercurio y la utilización de elementos de protección personal, la vigilancia estrecha de los niveles de mercurio en las mujeres gestantes y los potenciales efectos adversos en los neonatos, la determinación de los niveles de mercurio en agua, sedimientos y peces para consumo humano y la implementación de soluciones sistémicas, como la creación de centros para el proceso de amalgamación, que puedan controlar la exposición al mercurio (24).

\section{Agradecimientos}

A las bacteriólogas Aydé A. López y Eliana Sanguino y al personal que participó en las comisiones al río Inírida para la recolección de las muestras.

\section{Referencias}

1. Hamada R, Osame M. Minamata disease and other mercury syndromes. En: Chang LW, editor. Toxicology of metals. Boca Raton: CRC Lewis Publishers; 1996. p 346.

2. International Programme of Chemical Safety. Environmental health criteria 118. Inorganic mercury. Geneva: WHO-IPCS;1991.

3. León W. Visita de monitoreo a explotación minera en el río Inírida [preinforme]. Puerto Inírida: Servicio Seccional de Salud del Guainía, División de Saneamiento Ambiental; 1994.

4. Podlesky E, Ortiz JE, de García G. Determinación de trazas de metales en muestras biológicas y ambientales. Manual de procedimientos. Santafé de Bogotá: Instituto Nacional de Salud; 1992. p.15-38.

5. Hardman JG, Limbird LE. Toxicology. En: Goodman, Gilman, editores. The pharmacological basis of therapeutics. 9th edition. New York: McGraw-Hill Company; 1996. p.1658.

6. Vogelgesang J. Kalibo para calibración de métodos analíticos. Bruselas: Wogelgesang J;1994.

7. Cortés L, Rocha S, Ortiz JE. Determinación de cianuro y mercurio en aguas procedentes del departamento del Chocó. Santafé de Bogotá: Ministerio de Salud, Instituto Nacional de Salud; 1996.

8. Cortés L, Rocha S, Ortiz JE. Determinación de cianuro y mercurio en aguas procedentes del departamento de Nariño. Santafé de Bogotá: Ministerio de Salud, Instituto Nacional de Salud; 1996.

9. Cortés L, Rocha S, Ortiz JE. Determinación de cianuro y mercurio en aguas procedentes del departamento de Santander. Santafé de Bogotá: Ministerio de Salud, Instituto Nacional de Salud; 1996.
10. Cortés L, Rocha S, Ortíz JE. Determinación de cianuro y mercurio en aguas procedentes del departamento de Caldas. Santafé de Bogotá: Ministerio de Salud, Instituto Nacional de Salud; 1996.

11. Barbosa A, Boischio AAP. Exposição ao mercúrio orgânico em populações ribeirinhas do alto Madeira, Rondônia, 1991: resultados preliminares. Cad Saúde Pública 1993;9:155-60.

12. Couto RCSC, Camara VM, Sabroza PC. Intoxicação mercurial: resultados preliminares em duas áreas garimpeiras. Cad Saúde Pública 1988;4:301-15.

13. De Kom JFM, van der Voet GB, De Wolff FA. Mercury exposure of Maroon workers in the small scale gold mining in Suriname. Environ Res 1998;77:91-7.

14. Kehrig HA, Malm O, Akagi H, Guimaraes JRD, Torres JPM. Methylmercury in fish and hair samples from the Balbina reservoir, Brazilian Amazon. Environ Res 1998;77:84-90.

15. Malm O. Gold mining as a source of mercury exposure in the Brazilian Amazon. Environ Res 1998;77:738.

16. Boischio A, Barbosa A. Exposure to organic mercury in Riparian populations on the upper Madeira river, Rondonia, Brasil. Preliminary results. Cad Saúde Pública 1993;9:155-60.

17. Peixoto-Boischio AA, Cernichiari E. Longitudinal hair mercury concentration in riverside mothers along the upper Madeira river (Brazil). Environ Res 1998;77:7983

18. Olivero J, Mendonza C, Mestre J. Mercurio en cabello de diferentes grupos ocupacionales en una zona de minería aurífera en el norte de Colombia. Rev Saúde Pública 1995;29:376-9.

19. Olivero J, Solano B, Acosta I. Total mercury in muscle of fish from two marshes in goldfields, Colombia. Bull Environ Contam Toxicol 1998;61:182-7.

20. Olivero J, Solano B. Mercury in environmental samples from a waterbody contaminated by gold mining in Colombia, South America. Sci Total Environ 1998;217:83-9.

21. Morel FMM, Kraepiel AML, Amyot M. The chemical cycle and bioaccumulation of mercury. Annu Rev Ecol Syst 1998;29:543-66.

22. IPCS. Environmental Health Criteria 101. Methyl Mercury. Geneva: WHO-IPCS, 1990.

23. Sepúlveda J, López-Cervantes M, Frenk J, Goméz de León J, Lezana-Fernández MA, Santos-Burgoa C. Aspectos básicos de la vigilancia en salud pública para los años noventa. Salud Pública Mex 1994;36:7082.

24. Gasca-Alvarez AP. Environmental exposure to mercury in gold mining: health impact assessment in Guainía, Colombia. Rev Salud Pública 2000;2:233-50. 